\title{
Complete Atlases of Recalibrated Archival HST FOS Spectra of Quasars and AGN
}

\author{
S. Pesto, A. Koratkar, and E. Blitz
}

Space Telescope Science Institute, 3700 San Martin Drive, Baltimore, MD 21218, USA

\section{N. Evans}

Smithsonian Astrophysical Observatory, 60 Garden Street MS-27, Cambridge, MA 02138, USA

\section{Introduction}

The ionization structures of AGNs are determined by the specific ionization mechanisms and the shapes of the ionizing continua. The technique of using emission-line intensity ratios to classify objects and investigate their ionization mechanisms is enhanced significantly by combining high-quality UV and optical spectrophotometry. The HST archive is an excellent source of such data.

\section{Data Reduction: 'Average Inverse Sensitivity' Method}

The FOS spectra are recalibrated using the latest 'average inverse sensitivity' (AIS) calibration. This new method for flux calibrating FOS data (1) normalizes count data from all apertures to the $4^{\prime \prime} .3$ aperture to account for changes of aperture throughput as a function of the Optical Telescope Assembly focus; (2) corrects the data for time-dependent detector sensitivity degradation; and (3) scales the data to the white-dwarf reference scale. The AIS calibration method has been developed over a period of several years, with improvements applied progressively based on observed deficiencies and/or discrepancies of the existing calibrations. Indeed, the final AIS calibration incorporates corrections in the wavelength overlap regions of adjacent gratings derived based on inconsistencies discovered as a result of this study of over 1000 FOS spectra.

\section{Results of the Recalibration}

Figure 1 shows the differences between a recalibrated spectrum and the spectrum available from the $H S T$ data archive for a typical observation. For most preCOSTAR data, recalibration changes the photometry by $10-15 \%$. The most dramatic changes occur in the UV because of the difference between the old and new photometric reference scales.

Where possible, the recalibrated spectra have been combined carefully to produce high-quality, complete wavelength-coverage UV-optical spectra. Figure 2 shows a typical spectrum produced by combining multiple observations and 
Figure 1.

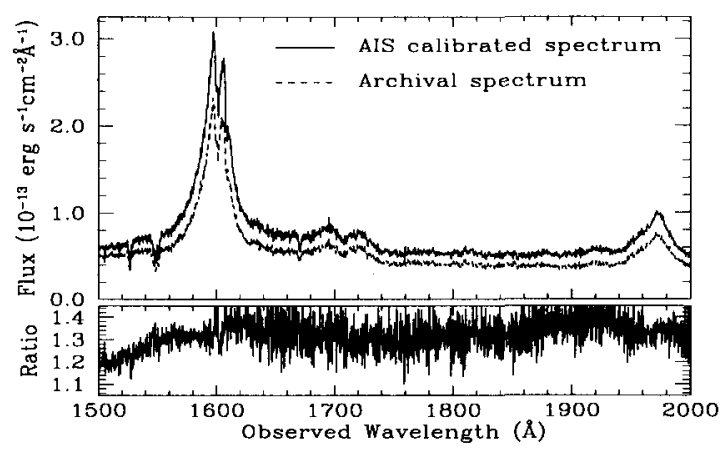

Figure 2.

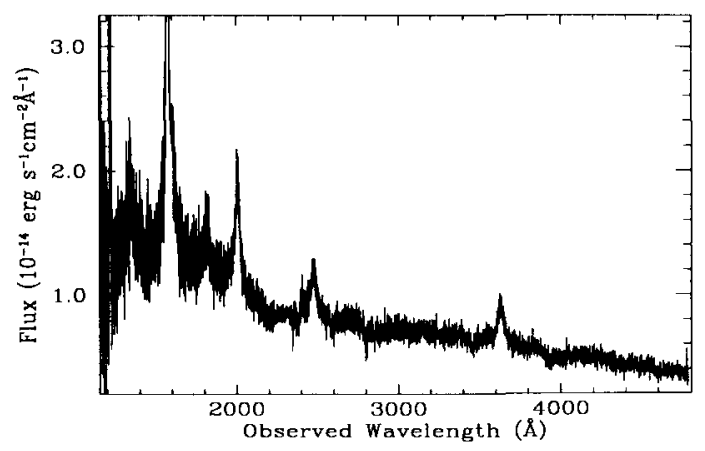

spectra obtained using several gratings. Before combining any spectra the observational consistency of the datasets is investigated, and effects such as aperture size dependence and time variability are considered carefully on a case by case basis.

Approximately $74 \%$ of datasets obtained as part of the same observation have fluxes in the grating overlap regions that are consistent to within $10 \%$. This is slightly worse than expected from observations of calibration standard stars, but routine (non-calibration) observations often employ less accurate target acquisition procedures, and scattered-light corrections may be less well determined for many AGN with strong continua.

The recalibrated spectra will be published shortly as atlases and will be available also in electronic form. We are currently in the process of measuring all of the UV and optical emission-line intensities and generating empirical diagnostic diagrams as ionization-mechanism discriminants. 